\title{
From Requirements Engineering to UML using Natural Language Processing - Survey Study
}

\author{
Omer Salih Dawood and Abd-El-Kader Sahraoui
}

\begin{abstract}
In the paper process of moving from software requirements to Unified Modeling Language (UML) diagrams has been studied. It shows the importance of this process and discusses many comparative studies in the field. A questionnaire related to the study was distributed worldwide to many research groups, academia, and industry to know the current status of using requirement management tools, knowledge of using UML in software development, frequently used UML diagrams, and the methodology used to generate UML diagrams from requirements. The paper emphasises that there is a need to do some important research in the area of requirements Natural Language processing (NLP) to obtain UML diagrams, and generalize process of using automatic or semi-automatic methodology to generate UML diagrams from requirements.
\end{abstract}

Index Terms-Requirement Engineering, Traceability, Requirements Management Tool, NLP, UML

\section{INTRODUCTION}

Requirements engineering is first step towards software development life cycle. It's aimed to collect and document requirements to be used in next steps. Bad collections and managing of requirement leads to software project failure or delivering projects on late. Success rate of software project is only $28 \%$ [21] .Many IT projects fail or are not delivered on time because of the lack of good requirements attributes. Handling the errors and problem earlier, better than handling it later and at low cost, requirements engineering is a challenge because of its critical steps and more work is needed to be done in research like documentation, verification and validation, traceability, and automatic moving from requirements to design step. To ensure that the requirements are properly taken into account, we need to trace these requirements to next steps that use these requirements. Another issue is generation of design from requirements, while preparing SRS document and moving to the next steps, some requirements are missed or not taken into account. Missing some requirements leads to customer dissatisfaction and incomplete product and this may take extra cost and time. The paper is consisted of the following sections. This section introduces the concepts of UML, Requirements Engineering, natural language processing and software engineering, and introduces the need of research in the area of generating diagrams from requirements. Section 2 concludes and compares the research work in the area of TEXT to UML, and introduces requirements management tool. Section 3 explain the Survey Study, and section 4 Discuss the survey results.

Published on January 1, 2017

Omer Salih Dawood is researcher in Sudan University of Science and Technology, Khartum, Sudan, and full time lecturer in Computer Science, College of Arts, Prince Sattam bin Abdulaziz University, KSA.

(e-mail: omercomail@gmail.com)

Abd-El-Kader Sahraoui is professor in LAAS-CNRS, Université de Toulouse, CNRS, UT2J, Toulouse, France.

\section{A. Unified Modelling Language (UML)}

UML is de facto standard for modeling of software. Object Management Group (OMG) was standardized UML in 1997 [16]. It is unification of three methods Booch, OMT, and OOSE.

According to [17] the UML has much type of diagrams, use case diagrams, and behavioral diagrams which include state diagram, activity diagram, sequence diagram, and collaboration diagram, and the other one is static diagrams like class diagram. It's a language for specifying, visualizing, constructing and documenting software systems. It's not just modeling software, many domains can be modeled UML like System Engineering, Process Modeling, and representing the organizational structures [18].

\section{B. Requirements Engineering}

According to SEI Requirements engineering confirms systematical and repeatable using of techniques ensure the completeness, consistency, and relevance of the system requirements [19]. It's very complex process because it involves the requester, developer, and author. The requesters know what they want but they don't know how to develop a system, while developer knows how to develop a system but they don't know what is the problem, the author tries to minimize communication gabs between requester and developer[19].There are two types of requirements, as follows:

A. User requirements: describe the services expected from system and constraints that the system should follow. It must be written in statements that the non-technical person can understand [20].

B. System requirements: describe more and deep 2details of user requirements. Software engineers analyze these requirements to know what exactly implement in the system. System requirements include both functional and non-functional requirements [20].

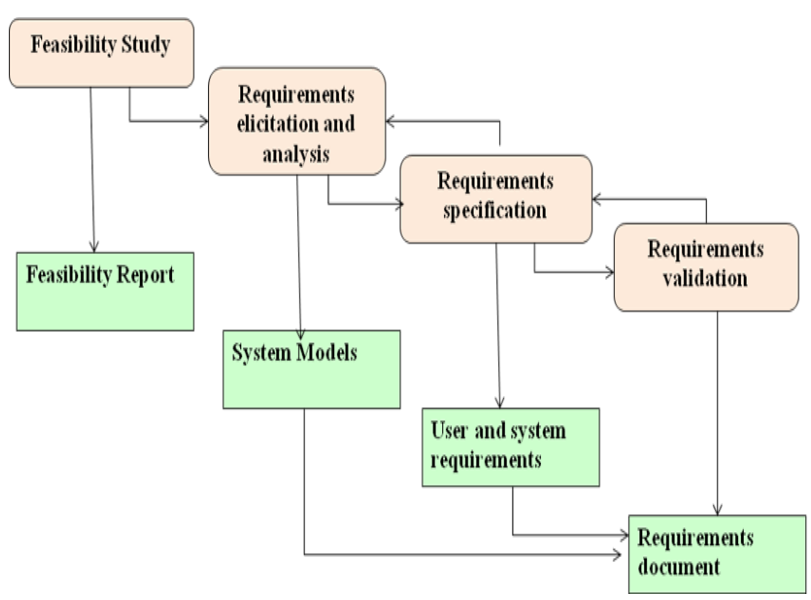

Fig. 1. Requirements Engineering Process. 


\section{Natural Language Processing and Software Engineering}

NLP is processing of human natural language automatically or semi-automatic. NLP is essentially multidisciplinary and related with linguistics [22].NLP and $\mathrm{SE}$ are both, the branches of computer science. In Software Development Life Cycle can be applied to every phase. There are textual artefacts in analysis and design phases like Requirement Document and Software Design Specification. Quality attributes like performance, feature, reliability, aesthetics, and perception are need to be assessed in NLP software [14]. Most of the requirements are written in natural language text and this causes many issues like ambiguity, specification issues, and incompleteness. These requirement statements need to be analyzed then we need to use natural language processing for that purpose because NLP provides many tools which help in linguistic analysis and helps in automated assistance [15]. Using NLP in RE is important because the requirement specification is written in cooperation between software house analyst and users and customers, and the customers will not sign contract if requirements are written in formal language [15].

\section{UML GENERATION FROM REQUIREMENTS USING NLP}

Priyanka and Rashmi [1] developed a tool for UML diagrams generation from requirements using NLP. The tool is known as (RAPID), which is used to analyse textual requirements, finding core concepts and its relationships, and finally generate UML diagram [1].

RAPID Consists of OpenNLP parser, RACE stemming algorithm, and Word Net, Domain Ontology module is used to improve the performance of concepts identification and Class Extraction Engine module receives the output of "concept extraction engine" module and applies different heuristic rules to generate both classes, relationship, and attributes [1].

Deva and Muhammad [2] developed a tool named as UML Model Generator from Analysis of Requirements (UMGAR) used to generate UML models from requirements using NLP tools, generated UML models like the Use-case Diagram, Analysis class model, Collaboration diagram and Design class model. They combined both Rational Unified Process (RUP) to specify objects, and ICONIX process that uses most a good NLP tools. There are two main components that composed UMGAR process architecture as follows:

1. Normalizing requirements component (NLP Tool Layer), used to identify requirements ambiguity and incompleteness and it consists of many sub components including Syntactic Reconstruction sub component uses 8 syntactic reconstruction rules.

2. Model Generator component used to generates many OO models from normalized requirements consists of Use-case Model Developer, and class model analysis and class model developer.

Andres and et al [3] developed a new approach and tool that interpret, organize, and manage requirements through application-specific ontologies and natural language processing. They used tool known as Natural Language Toolkit (NLTK) .The tool receives raw requirement text and performs segmentation to the entered text to obtain sentences, after that text will enter to tokenization process to tokenize text into words or punctuation character and normalize these tokens through stemming process, then perform par of speech tagging to identify the role of each word, entities can identify On top of POS tagging, then identify group tokens specially parsed words that represent the entity through chunking process, and finally recognize requirements[3]. NURI and et al [4] develop an approach to generate behavioral diagrams from user requirements they focused on both use case diagram and activity diagram. The developed tool named as RETRANS (REquirement TRANSformation). The approach receives the requirement and performs POS using Stanford POS Tagger, after that there are phases as follows:

Phase 1: requirement phase, first Phase is requirements keywords tracking used to detect the actor and use cases for the requirement. Phase 2: Use Case Diagram generation, the use case diagram library is used to generate use case diagram by connecting actors with related use case. Phase 3: Activity Diagram generation, after use case generation. The system automatically specifies all use cases that involved in the use case diagram, then it will generate the activity diagram by linking the components inside the class library (activity).

Prasanth Nakul [5] addresses the interdisciplinary between software engineering and natural language processing, and proposed methodology known as TextToUml to produce high-quality UML diagrams. The methodology consists of five diagrams as follows:

1- Define N.L. text quality parameters, and this includes classifying text to controlled language and uncontrolled language.

2- Identify level of noise and complexity of the text and classify sentences into simple, semi-complex, and complex.

3- Identify the type of diagram according to the description given in the text.

4- Determine UML diagram specification.

5- Derive UML specification with N.L text tuning to all available UML diagrams.

6- To generate UML diagrams, build interface between the ontology and application [5]

In [6] S.G. MacDonell and et al developed architecture of an autonomous requirements specification by using a natural language processing (NLP). They focused on the verification of requirements specification analysis.

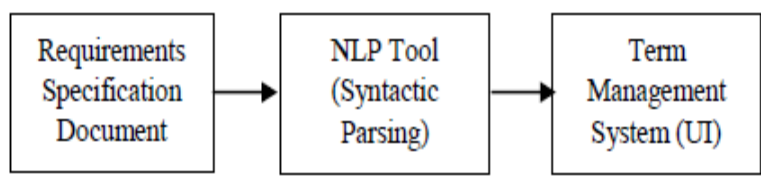

Fig. 2. System architecture.

As shown in figure (2) the system architecture consists of three modules as follows:

1- Tokeniser - reads requirements from a document

2- Parser - parses requirements sentence to extracts all unique noun terms.

3- Term management system - used to filter unimportant terms, classify remaining terms (function, entity, or attribute), and insert the object into a project knowledge base.

Subhash and et al [7] built a tool that analyzes requirement texts and builds model of the processed text represented in the semantic network. Their tool consists of 
two modules, NL analysis and diagram \& code generation [7].In the first module POS tagging is used to analyze and classify tokens, then the text understanding categorizes text into more further classes, as object, messages, verb, and etc to facilitate class generation process, Knowledge extraction module receives the output of previous phases to extract classes, attributes, and actors. Finally the UML diagrams will be generated. Based on generated UML diagrams, and extracted knowledge Second module generates code in language like java.

In Deva and Ratna [8] proposed a tool named as Static UML Model Generator from Analysis of Requirements (SUGAR) to produce static UML models from natural language. The SUGAR receives requirement texts and split complex sentences into simple sentences using syntactic reconstruction rules, then parse requirement document and generate parse tree by using Stanford Parser which the subjects and predicates are identified, actors mostly are subjects, and use cases are mostly the predicates, then identify the relationships between use cases and actors, then draw use case diagram. Class diagram can be generated by using the previous items and identify classes, methods, attributes, and relationships. Yasaman and et al [9] automated the process of generating package diagram in software design to facilitate design process and because process of moving from requirements engineering to design done by ad-hoc way. There are three components, first is static view generator - static ontology used to generate knowledge about the system. In the static ontology, classes with similar functionalities are grouped together. Second is dynamic view generator - dynamic view of ontology shows the interactions between the systems. Third is package diagram recommender that is used to receive the output of static and dynamic ontology generator to generate package diagram. A hierarchal clustering algorithm was used as core of packing solution. The classes are grouped together in same packages if they have higher number of communications and similar communication pattern between them [9].

Vibhu and et al [10] proposed a technique to generate high level class diagram from requirements, they implemented the approach into Functional Design Creation Tool (FDCT). They used heuristic rules and domain specific glossary to create the design. They developed Requirements Analysis Tool (RAT) that were used for restricts the requirement sentence, and perform lexical and semantic analysis on requirement document. RAT classifies requirements into six types which cover set of wide rage application requirement types. There are three phases approach for RAT to analyze requirements, in the first phase, requirements statement convert into a set of tokens with the user help to define glossaries. In the second phase the state machines are used for analysis of the requirement statements' syntax [10]. The third phase consists of semantic analysis with the help of domain specific ontology [10]. Sarita and Tanupriya [11] proposed an algorithm to automatically generate UML diagrams from user requirements after receiving requirements text, the text is tokenized and Pos tagger is used to perform lexical tagging, then extract verbs and objects as an activity, finally generate activity diagram.

To generate sequence diagram, after receiving requirements the plain text file is pre-processed, then the parser defines the structure of sentence [11] Imran and et al
[12] developed architecture to generate UML class diagram as in figure (3).

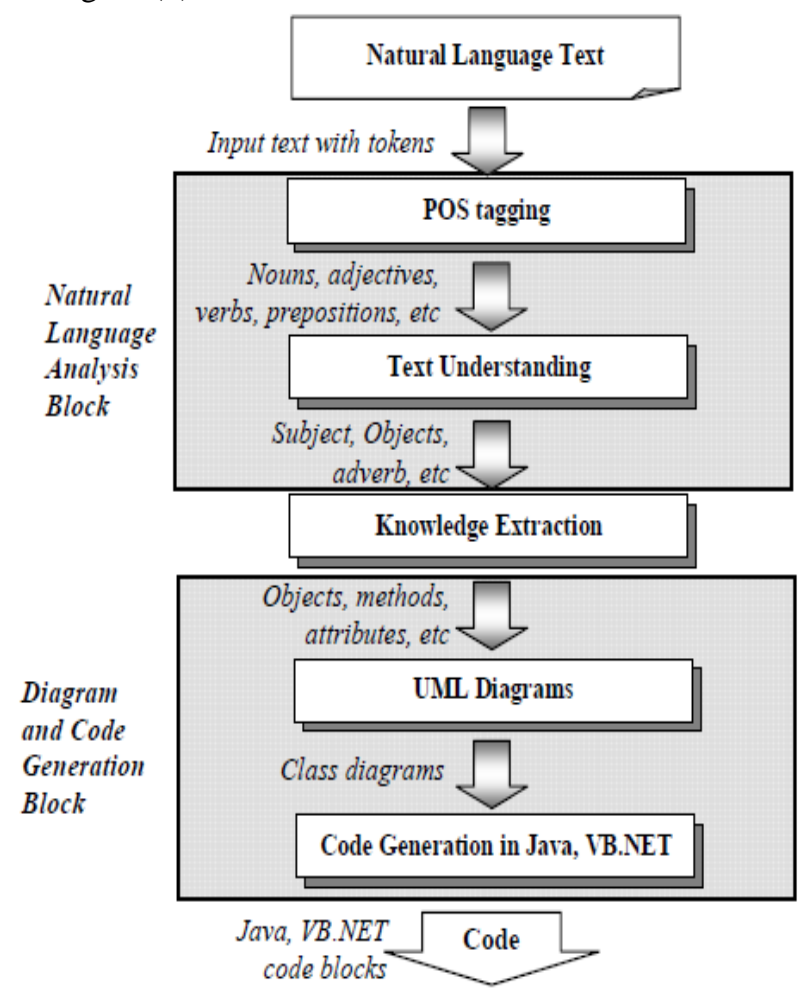

Fig. 3. Architecture of the designed system [12].

The architecture receives the requirements in text form and tokens the text, POS tagging receives the tokens to specify Nouns, adjectives, etc, after that the text understanding module specifies subjects, objects, etc, then knowledge extraction specifies objects, methods, attributes, etc, then the class diagram is generated and the code also can be generated with many languages in the last module [12].

Richa and et al [13] developed method to generate UML activity diagram, and sequence diagram they based their works on structured representation of requirements statements known as frame then using representation to generate activity and sequence diagrams. Requirements statements categorization is based on Grammatical Knowledge Patterns (English linguistics with the objective of understanding semantics of statements and extracting useful information) into Single category: Active or Passive voice, and Multiple categories: passive or active with one or more (Conjunction, Preposition, Precondition and Marker).There are four types of frame structure, active, passive, Conjunction between verbs with Passive Voice, and Preposition. The requirement statement is tokenized and belongs to specific frame, then the object, action, relationships can be identified [13]. 
TABLE I: Comparison Table.

\begin{tabular}{|c|c|c|c|c|}
\hline Study & Generated Diagrams & Main Components & Strengths & Weaknesses \\
\hline [1] & Class diagram & $\begin{array}{l}\text { RAPID Concept Extraction Engine+ } \\
\text { RAPID Class Extraction Engine + } \\
\text { some small components }\end{array}$ & $\begin{array}{l}\text { Deeply shows the details of class } \\
\text { elements generation }\end{array}$ & $\begin{array}{l}\text { Didn't generate class code }+ \text { only } \\
\text { concentrates in one diagram generation }\end{array}$ \\
\hline [2] & $\begin{array}{l}\text { use-case }+ \text { analysis } \\
\text { class } \\
\text { models+ } \\
\text { collaboration } \\
\text { diagram }\end{array}$ & $\begin{array}{lrr}\begin{array}{l}\text { Normalizing } \\
\text { component+ } \\
\text { component }\end{array} & \text { Model } & \text { requirements } \\
\text { Generator }\end{array}$ & Generates three UML diagrams & $\begin{array}{l}\text { Need some additives to generate more } \\
\text { diagrams }\end{array}$ \\
\hline [3] & Class Diagram & $\begin{array}{l}\text { Segmentation+ Tokenization + POS } \\
+ \text { entity and relation recognition }\end{array}$ & $\begin{array}{l}\text { Uses ontology to assist in } \\
\text { diagram generation and good in } \\
\text { identifying relationships }\end{array}$ & $\begin{array}{l}\text { Only class diagram generation without } \\
\text { code. Needs some additives to cover } \\
\text { many diagrams. }\end{array}$ \\
\hline [4] & $\begin{array}{l}\text { Use case and } \\
\text { Activity diagram }\end{array}$ & $\begin{array}{l}\text { NLP + use case library + activity } \\
\text { diagram library }\end{array}$ & Generate dynamic diagrams & $\begin{array}{l}\text { Some enhances are needed to generate } \\
\text { more diagrams }\end{array}$ \\
\hline [5] & Not specified & Many steps in algorithm & General diagram generation & Didn't show practical work \\
\hline [6] & Class diagram & $\begin{array}{l}\text { NLP tool + Term } \\
\text { Management } \\
\text { System }\end{array}$ & Extract complex sentence & $\begin{array}{l}\text { One diagram is few, need to generate } \\
\text { more }\end{array}$ \\
\hline [7] & class diagram & $\begin{array}{l}\text { Natural Language Analysis Block+ } \\
\text { Diagrams \& Code Generation Block }\end{array}$ & $\begin{array}{l}\text { Generate both static and } \\
\text { dynamic diagrams - use case and } \\
\text { class diagram and java class } \\
\text { code }\end{array}$ & $\begin{array}{l}\text { Some enhances are needed to generate } \\
\text { more static and dynamic diagrams }\end{array}$ \\
\hline [8] & $\begin{array}{l}\text { Usecase di- agram + } \\
\text { class diagram }\end{array}$ & $\begin{array}{l}\text { Use-case Model Generator + Class } \\
\text { Model Generator }\end{array}$ & $\begin{array}{l}\text { Generate both static and } \\
\text { dynamic diagrams - use case and } \\
\text { class diagram and java class } \\
\text { code }\end{array}$ & $\begin{array}{l}\text { Some enhances are needed to generate } \\
\text { more diagrams }\end{array}$ \\
\hline [9] & $\begin{array}{l}\text { Generate package } \\
\text { diagram }\end{array}$ & $\begin{array}{l}\text { Static Ontology } \\
\text { Generator + Dynamic Ontology } \\
\text { Generator }\end{array}$ & $\begin{array}{l}\text { Using ontology and Good in } \\
\text { grouping classes together }\end{array}$ & $\begin{array}{l}\text { One diagram is few, need to generate } \\
\text { more }\end{array}$ \\
\hline$[10]$ & class diagram & $\begin{array}{l}\text { RAT }+ \text { Tokens }+ \text { heuristic rules }+ \\
\text { UML creator module }\end{array}$ & $\begin{array}{l}\text { Generate a high-level class } \\
\text { diagram in a good methodology }\end{array}$ & $\begin{array}{l}\text { One diagram is few, need to generate } \\
\text { more+didn't generate classes code }\end{array}$ \\
\hline$[11]$ & $\begin{array}{l}\text { Activity diagram }+ \\
\text { sequence diagram }\end{array}$ & $\begin{array}{l}\text { Activity: Sentence splitter + POS } \\
\text { tagger + verb and object extractor. } \\
\text { Sequence: pre-processing + parser + } \\
\text { additional information identifier + } \\
\text { adding conditions }\end{array}$ & $\begin{array}{l}\text { Generation both activity and } \\
\text { sequence diagrams in good } \\
\text { manner }\end{array}$ & $\begin{array}{l}\text { Concentrates only in some dynamic } \\
\text { diagram }\end{array}$ \\
\hline [12] & Class diagram & $\begin{array}{l}\text { Natural Language Analysis } \\
\text { Block[POS tagging + Text } \\
\text { Understanding]+Knowledge } \\
\text { Extraction +Diagram } \\
\text { and Code Generation Block [ UML } \\
\text { diagrams]+ Code Generation }\end{array}$ & $\begin{array}{l}\text { Generate UML class diagram in } \\
\text { a good way with class's code. }\end{array}$ & $\begin{array}{l}\text { Only one static class diagrams are } \\
\text { generated, need more enhancement to } \\
\text { generate more classes }\end{array}$ \\
\hline [13] & $\begin{array}{l}\text { Activity diagram }+ \\
\text { sequence diagram }\end{array}$ & $\begin{array}{l}\text { Stanford POS tagger }+ \text { Frame } \\
\text { structure(Active } \text { Voice, Passive } \\
\text { Voice, Conjunction between Verbs } \\
\text { with Passive Voice, and Preposition) }\end{array}$ & $\begin{array}{l}\text { Classify statements into simple } \\
\text { and complex statements. Frame } \\
\text { is a good idea to generate both } \\
\text { activity and sequence diagram }\end{array}$ & $\begin{array}{l}\text { They assume that no redundancy and } \\
\text { ambiguity. Need some enhancement to } \\
\text { cover more diagrams }\end{array}$ \\
\hline
\end{tabular}

\section{Methodology}

The research aimed to improve the process of generating UML diagrams from requirements, many related work are reviewed firstly to specify the weaknesses and what is the problem in this area, to know the range of using requirements in organized way and how the organizations generate UML from requirements, to know all these a Questionnaire was developed by consulting many computer science professors and distributed worldwide to many IT companies, universities, and research groups to ensure the questionnaire covers wide range, and allow to obtain good and common result. Both academia and industry that related with software and system engineering filled the questionnaire, we expected more than 100 persons will fill the questionnaire but only around 92 persons filled the questionnaire, and good results obtained and the research achieve the objectives. The questionnaire was distributed and filled during the period from 01 November to 01 December 2016. The questionnaire has many sections each one concentrates to specific questions, there is section to now the usage of requirement management tools, section to know the preferences of UML diagrams generations from requirements, section to know most UML diagrams used.

This questionnaire aims to answer the following questions:

1. What is familiarity of Formal methods, SADT (Structured Analysis and Design Technique), and OMT (Object Modelling Technique)?

2. Do the organizations \system engineers use a systematic way to gather and document requirements and familiarity in using requirements management tool?

3. How organizations \system engineers generate UML diagrams from requirements?

4. Are organizations $\backslash$ system engineers need tools/techniques that facilitate the process of moving from requirements engineering stage to software design stage?

5. The order of using UML diagrams - what is most used and needed UML diagrams.

\section{RESULTS AND DISCUSSIONS}

The questionnaire was distributed worldwide and filled by 92 respondents from academia, industry, or both we found that around $25 \%$ weren't familiar with Formal 
methods, SADT (Structured Analysis and Design Technique), and OMT (Object Modelling Technique), and around $75 \%$ familiar with one or more from these concepts. There are $92 \%$ familiar with basic UML concepts. The survey found that $69 \%$ is using a systematic methodology for collecting requirements, while $31 \%$ collect requirements without systematic methodology, and around $67 \%$ of the organizations follow Software Development Life Cycle to develop systems. Regarding knowledge of using requirement management tools we found that $17.8 \%$ has strong knowledge, while $45.6 \%$ has medium knowledge, and 36.7 has poor knowledge. This means that there is need to encourage the organizations \system engineers to use requirement management tools. It is very important to generate UML diagrams from requirements by aiding of tool to ensure all requirements are covered into design then obtain high quality software, as shown in figure (4), $40.2 \%$ organizations $\backslash s y s t e m$ engineers generate UML diagrams manually, while $23.9 \%$ generate UML diagrams by semiautomatic methodology, $13 \%$ generate UML diagrams by automatic method, and $22.8 \%$ didn't generate UML diagrams from requirements. Manual process of generation of UML from requirements needs some enhancement to become automatic or semi-automatic.

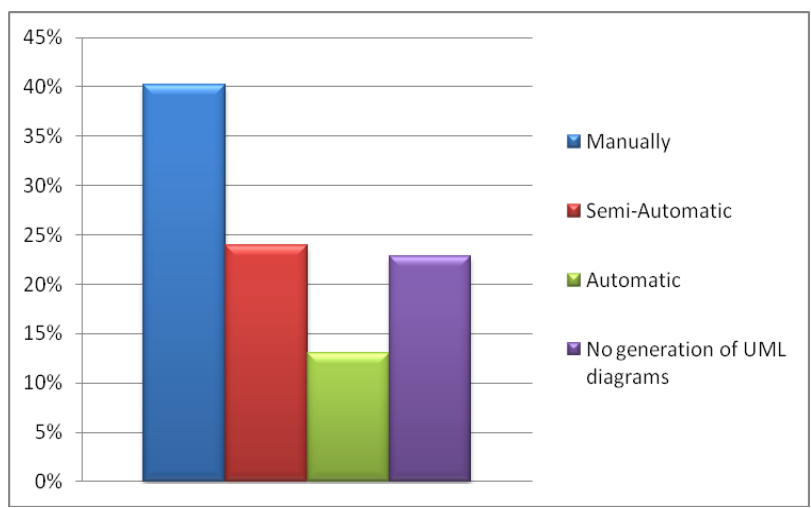

Fig. 4. Methodology of UML generation.

UML has many types of diagrams each one has specific role. Figure (4) shows the order of using UML diagrams, the use case diagram, class diagram, sequence diagram, and activity diagram has high percentage use respectively. It's necessary to help generation of these diagrams according to the order of usage.

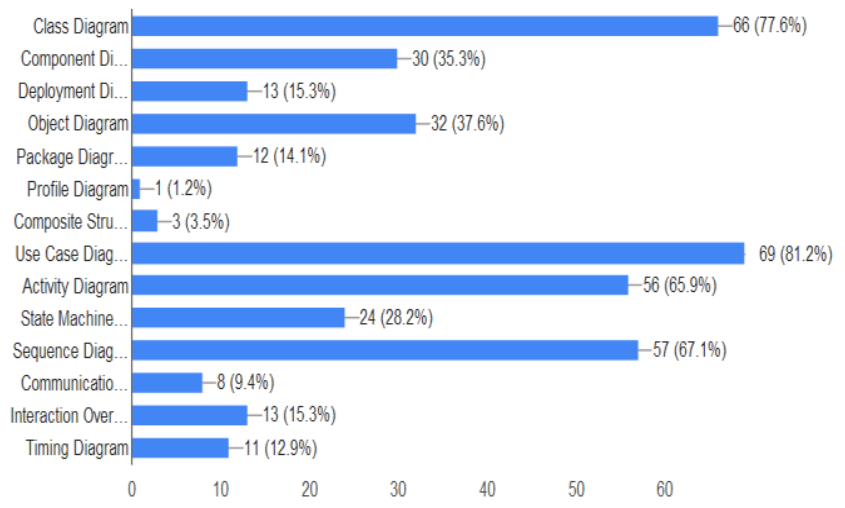

Fig. 5. Percentage of using UML diagrams.

\section{CONCLUSION}

The paper reviewed the current status of using Natural language processing in software engineering to process the software requirements to generate UML diagrams. The paper deeply studied many research in this area and made a comparison between them and identified each weaknesses and strength and scope for improvement.

A Questionnaire was distributed worldwide about using UML, requirement management tools, and how the software engineers generate UML diagrams from requirements. The main purpose of this questionnaire was to enhance software quality and minimize the design time, cost, and error with reducing human intervention in the design phase through improving the process of generating UML diagrams from requirements using NLP.

We found that only around $13 \%$ of users/organizations generate UML diagrams automatically and around 23.9 using semi-automatic way to generate UML diagrams, while $40.2 \%$ generate UML diagrams manually, and this means that there is need to minimize this percentage by more studies and enhancing in UML generation from requirements automatically or semi automatic way.

\section{REFERENCES}

[1] More, Priyanka, and Rashmi Phalnikar. "Generating UML Diagrams from Natural Language Specifications." International Journal of Applied Information Systems, Foundation of Computer Science 1.8 (2012)

[2] Deeptimahanti, Deva Kumar, and Muhammad Ali Babar. "An automated tool for generating UML models from natural language requirements." Proceedings of the 2009 IEEE/ACM International Conference on Automated Software Engineering. IEEE Computer Society, 2009.

[3] Arellano, Andres, Edward Carney, and Mark A. Austin. "Natural Language Processing of Textual Requirements." The Tenth International Conference on Systems (ICONS 2015), Barcelona, Spain. 2015.

[4] Kamarudin, Nuri Jazuli, Nor Fazlida Mohd Sani, and Rodziah Atan. "AUTOMATED TRANSFORMATION APPROACH FROM USER REQUIREMENT TO BEHAVIOR DESIGN." Journal of Theoretical and Applied Information Technology 81.1 (2015): 73.

[5] Yalla, Prasanth, and Nakul Sharma. "Utilizing NL Text for Generating UML Diagrams." Proceedings of the International Congress on Information and Communication Technology. Springer Singapore, 2016.

[6] MacDonell, Stephen G., Kyongho Min, and Andy M. Connor "Autonomous requirements specification processing using natural language processing." arXiv preprint arXiv:1407.6099 (2014).

[7] Shinde, Subhash K., Varunakshi Bhojane, and Pranita Mahajan. "Nlp based object oriented analysis and design from requirement specification."International Journal of Computer Applications 47.21 (2012)

[8] Deeptimahanti, Deva Kumar, and Ratna Sanyal. "An innovative approach for generating static UML models from natural language requirements."International Conference on Advanced Software Engineering and Its Applications. Springer Berlin Heidelberg, 2008.

[9] Amannejad, Yasaman, et al. "From requirements to software design: An automated solution for packaging software classes." Information Reuse and Integration (IRI), 2014 IEEE 15th International Conference on. IEEE, 2014.

[10] Sharma, Vibhu Saujanya, et al. "Extracting high-level functional design from software requirements." 2009 16th Asia-Pacific Software Engineering Conference. IEEE, 2009.

[11] Gulia, Sarita, and Tanupriya Choudhury. "An efficient automated design to generate UML diagram from Natural Language Specifications." Cloud System and Big Data Engineering (Confluence), 2016 6th International Conference. IEEE, 2016. 
[12] Bajwa, Imran Sarwar, Ali Samad, and Shahzad Mumtaz. "Object oriented software modeling using NLP based knowledge extraction." European Journal of Scientific Research 35.01 (2009) 22-33.

[13] Sharma, Richa, Sarita Gulia, and K. K. Biswas. "Automated generation of activity and sequence diagrams from natural language requirements."Evaluation of Novel Approaches to Software Engineering (ENASE), 2014 International Conference on. IEEE, 2014.

[14] Yalla, Prasanth, and Nakul Sharma. "Integrating Natural Language Processing and Software Engineering." International Journal of Software Engineering and Its Applications 9.11 (2015): 127-136.

[15] Lash, Alex, Kevin Murray, and Gregory Mocko. "Natural language processing applications in requirements engineering." ASME 2012 International Design Engineering Technical Conferences and Computers and Information in Engineering Conference. American Society of Mechanical Engineers, 2012.

[16] Hilliard, Rich. "Using the UML for architectural description." International Conference on the Unified Modeling Language. Springer Berlin Heidelberg, 1999.

[17] Yang, Kann-Jang, and Rob Pooley. "Process modelling to support the Unified Modelling Language." Computer Software and Applications Conference, 1997. COMPSAC'97. Proceedings., The Twenty-First Annual International. IEEE, 1997.

[18] Fitsilis, Panos, Vassilis C. Gerogiannis, and Leonidas Anthopoulos. "Role of unified modelling language in software development in Greece?? results from an exploratory study." IET Software 8.4 (2014): 143-153.

[19] SEI. (November 2016). A Framework for Software Product Line Practice, available: http://www.sei.cmu.edu/productlines/frame_report/req_eng.htm

[20] S. Guanda, "Requirements Engineering: elicitation techniques" M.S. thesis, Dept. Technology, Math, and computer science, Univ. of WEST , Sweden , 2008

[21] Muqeem, Mohd, and Mohd Rizwan Beg. "Validation of requirement elicitation framework using finite state machine." Control, Instrumentation, Communication and Computational Technologies (ICCICCT), 2014 International Conference on. IEEE, 2014.

[22] Ann Copestake, "Natural Language Processing," Lecture Synopsis , 2004 